\title{
Adaptive timing of motor output in the mouse: the role of movement oscillations in eyelid conditioning
}

\section{Selmaan N. Chettih, Samuel D. McDougle, Luis I. Ruffolo and Javier F. Medina*}

Department of Psychology, University of Pennsylvania, Philadelphia, PA, USA

Edited by:

Agnes Gruart, University Pablo de Olavide, Spain

\section{Reviewed by:}

S. K. E. Koekkoek, Erasmus MC, Netherlands

Raudel Sánchez-Campusano,

Universidad Pablo de Olavide, Spain

\section{*Correspondence:}

Javier F. Medina, Department of

Psychology, University of

Pennsylvania, Solomon Labs Building,

3720 Walnut Street, Philadelphia, PA

19104, USA.

e-mail: jmed@psych.upenn.edu

\begin{abstract}
To survive, animals must learn to control their movements with millisecond-level precision, and adjust the kinematics if conditions, or task requirements, change. Here, we examine adaptive timing of motor output in mice, using a simple eyelid conditioning task. Mice were trained to blink in response to a light stimulus that was always followed by a corneal air-puff at a constant time interval. Different mice were trained with different intervals of time separating the onset of the light and the air-puff. As in previous work in other animal species, mice learned to control the speed of the blink, such that the time of maximum eyelid closure matched the interval used during training. However, we found that the time of maximum eyelid speed was always in the first $100 \mathrm{~ms}$ after movement onset and did not scale with the training interval, indicating that adaptive timing is not accomplished by slowing down (or speeding up) the eyelid movement uniformly throughout the duration of the blink. A new analysis, specifically designed to examine the kinematics of blinks in single trials, revealed that the underlying control signal responsible for the eyelid movement is made up of oscillatory bursts that are time-locked to the light stimulus at the beginning of the blink, becoming desynchronized later on. Furthermore, mice learn to blink at different speeds and time the movement appropriately by adjusting the amplitude, but not the frequency of the bursts in the eyelid oscillation.
\end{abstract}

Keywords: eyeblink, cerebellum, interstimulus interval, invariance, learning

\section{INTRODUCTION}

Timing is everything. The meaning of this expression is perhaps most obvious in motor control, where a delay of just few milliseconds can make the difference between hitting a home-run or striking out (Williams and Underwood, 1986; Gray, 2002). To achieve such remarkable precision, we must learn to estimate temporal contingencies accurately despite living in an ever-changing world, and adjust the timing of our movements accordingly. For example, good hitters are capable of adapting their swing depending on pitch type, often taking into account external factors that affect the speed of the baseball, like wind, humidity, temperature, or altitude (Williams and Underwood, 1986; Gray, 2002; Fortenbaugh, 2011).

In the laboratory, adaptive timing has been studied extensively using the eyelid conditioning task (Gormezano et al., 1983). Subjects learn to blink in response to a conditioned stimulus (CS), like a light, that precedes and is repeatedly paired with an air-puff directed at the eye. Previous work in a variety of animal species has demonstrated that the timing of the conditioned eyelid response is adjusted to match the interstimulus interval (ISI): maximum eyelid closure in "test-trials" without an air-puff occurs at the time when the air-puff is normally delivered, regardless of the particular ISI used during training (Boneau, 1958; Mauk and Ruiz, 1992; Domingo et al., 1997; Freeman et al., 2003; Koekkoek et al., 2003). Measuring the latency to maximum eyelid closure, however, does not provide information about the particular trajectory that the eyelid takes from open to closed.
To really understand the control signals used to achieve timing, it is necessary to characterize the kinematics of the movement and examine how it unfolds in time. Most experimental work supports the notion that the timing of the conditioned eyelid response is achieved by adjusting the speed, and to a lesser extent the onset, of the movement (Boneau, 1958; Levey and Martin, 1968; Mauk and Ruiz, 1992; Domingo et al., 1997; Freeman et al., 2003; Koekkoek et al., 2003). Although a full description of blink kinematics at different ISIs is still lacking, this type of trajectory has been modeled as being timescale-invariant (Grossberg and Schmajuk, 1989; Lepora et al., 2007): the timing of movement is adaptively adjusted by slowing down (or speeding up) the entire movement trajectory, from beginning to end, by a constant factor. For many movements, the underlying rules of adaptive timing are known, and appear to be compatible with the timescale invariance hypothesis (Schmidt and Lee, 2005), but see (Gentner, 1987). In contrast, and despite the existing wealth of data about adaptive timing in the eyelid conditioning task, the rules for adjusting the conditioned eyelid trajectory remain poorly understood.

One source of complication is that most studies have examined the timing of the conditioned eyelid movement by averaging trajectories over many trials (Levey and Martin, 1968; Mauk and Ruiz, 1992; Domingo et al., 1997; Freeman et al., 2003; Koekkoek et al., 2003), masking the fact that individual blinks are not smooth. As documented by work in a variety of animal species, including human (Marquis and Porter, 1939), rabbit (Gruart et al., 2000), cat (Domingo et al., 1997), ferret (Ivarsson and Svensson, 2000), 
guinea pig (Gruart et al., 2000), rat (Gruart et al., 2000), and mouse (Koekkoek et al., 2002), conditioned eyelid movements display prominent oscillations, accelerating and decelerating in brief bursts which can be detected in the electromyographic (EMG) activity of the orbicularis oculi muscle (Gruart et al., 1995, 2000; Trigo et al., 1999; Ivarsson and Svensson, 2000). In theory, adaptive timing could be achieved by modulating the amplitude, rate, and/or number of these bursts, in ways that could make the movement timescale-invariant or not. Which control strategy is actually implemented by the brain is not known.

In this study we characterize the kinematics of the conditioned eyelid response in mice trained to different ISIs. Our results demonstrate that the conditioned eyelid movement consists of two phases: an initial acceleration phase from movement onset to maximum eyelid speed that is highly stereotyped and has the same duration regardless of ISI, and a subsequent deceleration phase from maximum eyelid speed to the moment of maximum eyelid closure, whose duration is adjusted and is proportional to the ISI. The resulting conditioned eyelid trajectory is not timescaleinvariant, but nonetheless it achieves maximum eyelid closure around the time of the particular interval used during training. Single-trial analyses reveal that these adjustments to the timing of the conditioned eyelid response are made by neural mechanisms that modulate the amplitude of the underlying bursts of movement, but not the frequency at which they occur.

\section{RESULTS}

Although there have been sporadic accounts of adaptive timing during eyelid conditioning in mice (Koekkoek et al., 2003; Van Der Giessen et al., 2008; Boele et al., 2010), no systematic study of the eyelid trajectories has been carried out. Thus, we begin by characterizing in full detail the kinematic properties of the conditioned eyelid response in mice trained at different ISIs. Light was used as the CS because it did not cause startle-responses, unlike the more commonly used auditory tone CS (Boele et al., 2010). In all our experiments the light stimulus was visible by the two eyes, the puff was delivered to the left eye, and conditioned responses were measured exclusively by examining the left eye. To allow for unbiased comparisons across different ISI conditions, analysis was performed on test sessions that began after asymptotic performance had been achieved, and by examining conditioned eyelid responses only on CS-alone trials in which no air-puff is delivered (see Materials and Methods). As shown in Table 1, mice trained with 175 or $250 \mathrm{~ms}$ intervals showed high-levels of conditioning and generated conditioned eyelid responses reliably, while performance for mice trained with 325 or $400 \mathrm{~ms}$ intervals exhibited greater variance.

\section{GENERAL KINEMATIC PROPERTIES OF THE CONDITIONED EYELID MOVEMENT}

Conditioned eyelid responses were adaptively timed. As ISI increased from 175 to $400 \mathrm{~ms}$, the learned blinks became smaller in amplitude, and latency to maximum eyelid closure increased adaptively to match the ISI used during training (Table 1; Figure 1A; $p<0.001$ confirmed with sequential $t$-tests between the different ISI conditions). Responses appeared to become broader as the ISI was lengthened, rather than simply translated later in time. In other words, over the range of ISIs in Figure 1, mice appear to achieve precise timing by regulating the velocity, but not the onset latency of the eyelid movement (Figures 1B,C). As shown in Figure 1C, maximum velocity and maximum eyelid closure were strongly correlated in individual trials $(R$-values ranged from 0.62 to 0.72 for the different conditions), and the average of both kinematic parameters decreased with ISI ( $p<0.001$ confirmed with sequential $t$-tests). The regression lines overlapped for ISIs in the range $250-400 \mathrm{~ms}$, suggesting that the same relationship between maximum velocity and maximum eyelid closure is applicable across these different conditions. The relationship was still present in mice trained with the 175-ms ISI (red regression line), but in this group, conditioned eyelid responses of comparable maximum closure reached higher speeds than in mice trained with the other ISIs.

Table 1 | Kinematic properties of the average conditioned eyelid response for each of the mice in this study.

\begin{tabular}{lllll}
\hline ISI (ms) & \% CR & Max closure (FEC) & Time max closure (ms) & Max vel (FEC/s) \\
\hline 175 & $81 \pm 4$ & $0.49 \pm 0.17$ & $237 \pm 74$ & $16 \pm 7.0$ \\
& $87 \pm 5$ & $0.59 \pm 0.18$ & $235 \pm 76$ & $18 \pm 6.5$ \\
& $93 \pm 4$ & $0.60 \pm 0.16$ & $237 \pm 53$ & $9.2 \pm 3.7$ \\
250 & $83 \pm 6$ & $0.37 \pm 0.13$ & $275 \pm 71$ & $9.1 \pm 3.7$ \\
& $92 \pm 7$ & $0.42 \pm 0.17$ & $274 \pm 65$ & $15 \pm 6.1$ \\
& $90 \pm 4$ & $0.72 \pm 0.20$ & $365 \pm 114$ & $10 \pm 4.0$ \\
325 & $85 \pm 5$ & $0.44 \pm 0.13$ & $284 \pm 79$ & $11 \pm 4.8$ \\
& $79 \pm 7$ & $0.43 \pm 0.14$ & $397 \pm 122$ & $9.0 \pm 4.8$ \\
& $72 \pm 6$ & $0.43 \pm 0.16$ & $368 \pm 90$ & $8.5 \pm 5.1$ \\
400 & $56 \pm 14$ & $0.37 \pm 0.16$ & $367 \pm 141$ & $9.1 \pm 4.3$ \\
& $95 \pm 4$ & $0.44 \pm 0.14$ & $436 \pm 144$ & $9.7 \pm 3.7$ \\
& $52 \pm 10$ & $0.35 \pm 0.17$ & $390 \pm 144$ & $9.2 \pm 5.9$ \\
& $88 \pm 9$ & $0.34 \pm 0.18$ & $411 \pm 114$ & $9.0 \pm 4.7$ \\
\hline
\end{tabular}

Error ranges for "\% CR" are SD over individual sessions; all other error ranges are SD over all trials. CR, conditioned response; FEC, fraction eyelid closure. 

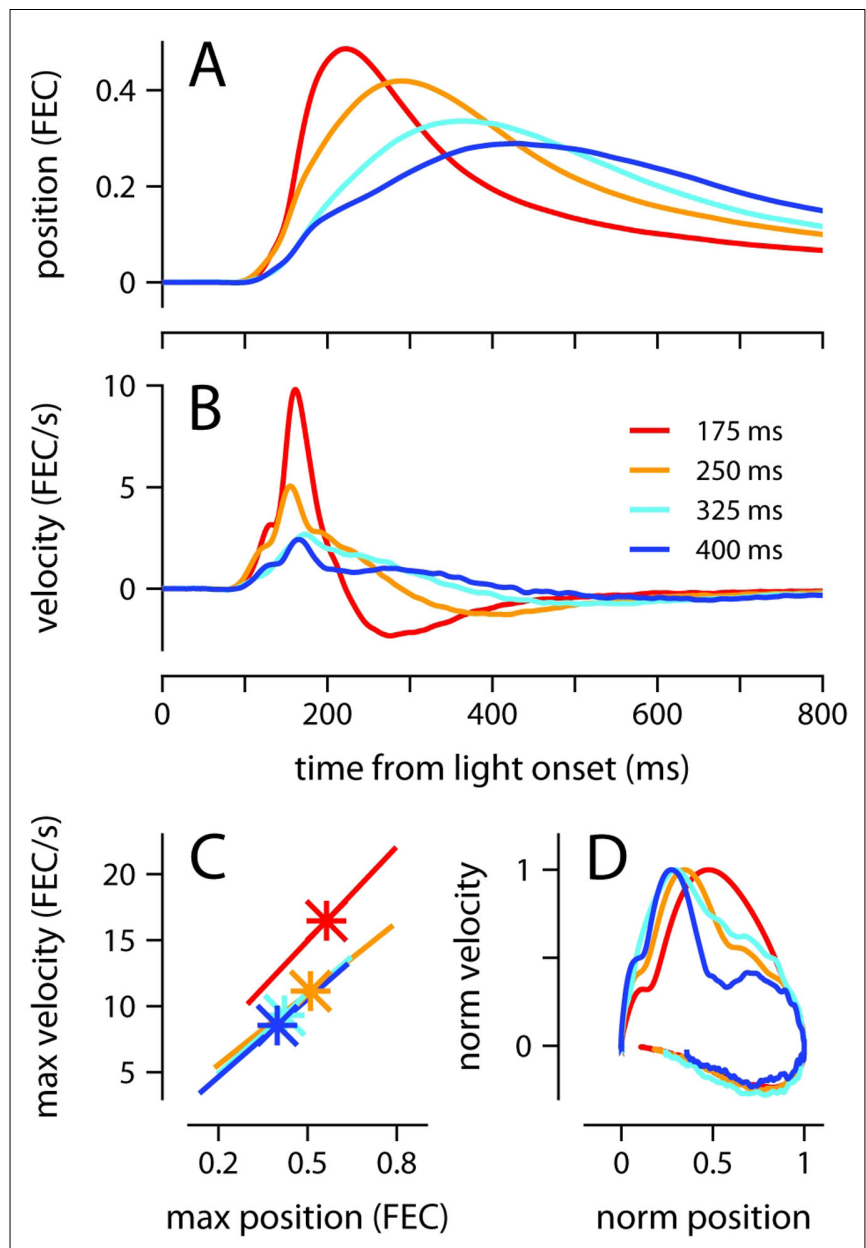

FIGURE 1 | Kinematic properties of the conditioned eyelid movement. (A) Average eyelid position, and (B) average eyelid velocity for mice trained with four different ISIs. (C) Asterisks indicate the average maximum position and maximum velocity for each ISI. X-range of the linear regression lines is mean of position maxima \pm std across all trials for a particular ISI. (D) Normalized phase plots for the data shown in $(\mathbf{A}, \mathbf{B})$. Color code for all plots indicates trained ISI and is shown in (B). FEC, fraction eyelid closure.

The broadening of the conditioned response as the ISI was lengthened was not a simple timescale-invariant stretching of the movement. This can be appreciated visually by comparing the peaks of the traces in Figures 1A,B. The time of maximum eyelid closure scales proportionally with the ISI whereas the time of maximum eyelid velocity remains relatively constant. Indeed, the normalized phase plots in Figure 1D do not overlap each other, and clearly show that for example, maximum velocity was attained when the eyelid had closed $50 \%$ of the size of the full conditioned response in the 175-ms group (red line), but only $25 \%$ in the 400 -ms group (blue line). The same asymmetric pattern in the temporal scaling of eyelid position vs. velocity was clearly visible when the kinematic data from individual mice was analyzed separately and normalized to emphasize temporal structure over differences in amplitude (Figures 2A,B). As shown in the probability density plots of Figures 2C,D, this effect was not an artifact of averaging many conditioned eyelid responses: the time of maximum eyelid closure in individual trials became more variable with longer ISIs, but was also clearly shifted later in time (Figure 2C). In stark contrast, most eyelid movements reached maximum velocity between 150 and $180 \mathrm{~ms}$ after light onset for all mice, regardless of the ISI used during training, although the range of peak-times did appear to extend later in time as ISI increased (Figure 2D).

Because Figures 1 and 2 clearly demonstrate that conditioned eyelid responses are not timescale-invariant, understanding the rules of response timing necessitates moving beyond traditional latency-to-peak analyses to a richer understanding of the temporal evolution of the movement kinematics. This is the focus of the next sections.

\section{CONDITIONED EYELID MOVEMENTS BEGIN WITH TWO TIME-LOCKED BURSTS OF ACCELERATION}

The mean velocity of the eyelid movement exhibited a stereotyped profile in the first $200 \mathrm{~ms}$ after the presentation of the light regardless of ISI (Figures 2B and 3), with onset occurring around $100 \mathrm{~ms}$, a small bump or local peak prior to $150 \mathrm{~ms}$, and a second peak between 150 and $180 \mathrm{~ms}$, which was always the velocity maximum. The velocity then ramped down with a somewhat idiosyncratic trajectory, remaining positive (eyelid closing) for a duration that was inversely related to ISI. This stereotyped profile was strikingly similar for all trained ISIs, and was not an artifact of averaging over multiple recording sessions because the same pattern was clearly observed in individual sessions (Figures 3A,B).

To investigate the underlying cause of this stereotyped profile, which represents the average over many conditioned eyelid movements, we examined eyelid velocity on individual trials. Figures 3C,D show eyelid velocity and position traces from three example trials taken from the mouse in Figure 3B. The velocity profile in the first $200 \mathrm{~ms}$ of individual trials exhibited an underlying oscillation, with multiple peaks (Figure 3E) separated by a mean interpeak-interval of $\sim 30 \mathrm{~ms}$ (Figure $3 \mathrm{~F}$ ), with many separated by exactly $29 \mathrm{~ms}$. We found many trials in which the oscillation began at the same time and remained synchronized and in phase for the first $200 \mathrm{~ms}$ (Figure 3C), accounting for the two peaks in the velocity profile that appear at the same location on mean behavior (Figure 3B). Though the oscillation in eyelid velocity remains for the entire duration of the movement, it appears to become desynchronized across trials after $200 \mathrm{~ms}$ from the time of light onset (Figure 3C).

This effect, which suggests that the eyelid accelerates and decelerates in discrete bursts which are precisely time-locked early in the movement, and less so later on, was further examined as follows (see Materials and Methods for details): local peaks and troughs in the oscillatory eyelid velocity were detected in individual trials, and this information was used to compute the difference between the probability of finding a peak and the probability of finding a trough as a function of time, for each mouse separately (Figure 3G), and also averaged across individuals trained with the same ISI (Figure 3H). There were slight differences between mice, but there is a clear tendency for peaks of eyelid velocity to cluster at two separate times in the first $200 \mathrm{~ms}$ after light onset. The location of the two clusters did not appear related to the trained ISI (Figures 3G,H). Although the number of peaks and troughs 

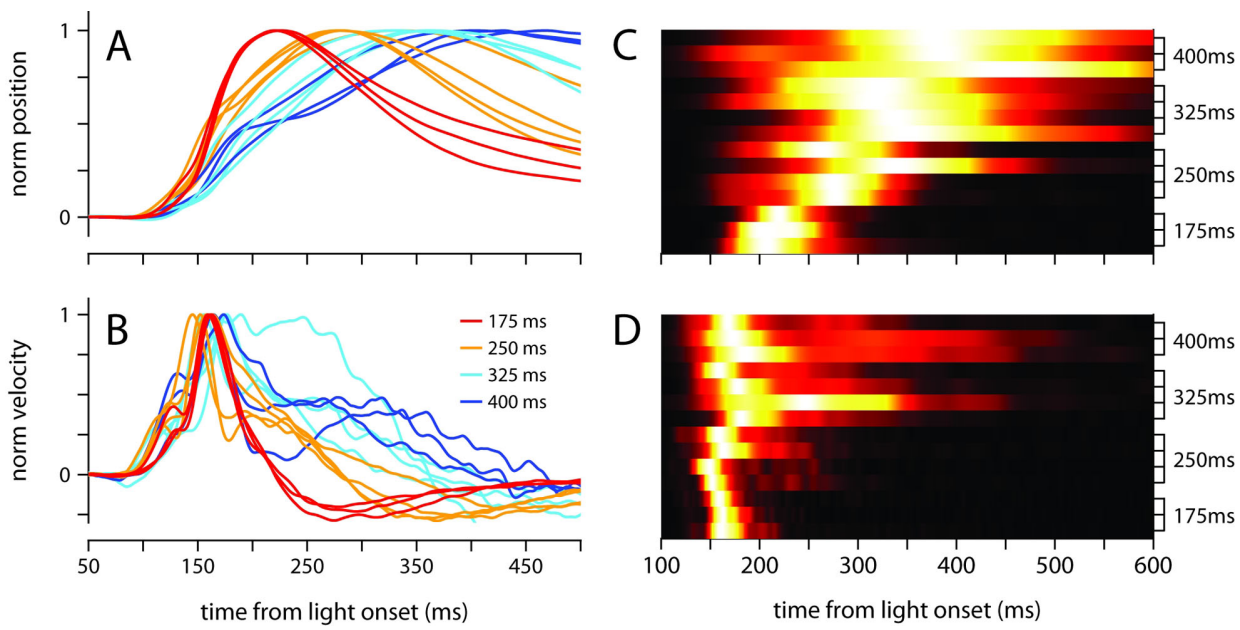

FIGURE 2 |Temporal profile of the conditioned eyelid movement in individual mice. (A) Normalized average position, and (B) normalized average velocity, of each mouse's conditioned eyelid responses. Color code indicates trained ISI and is shown in (B). (C) Probability density functions for latency to

maximum eyelid closure, and (D) latency to maximum velocity. Each row of the heat map contains the probability density function for an individual mouse, with trained ISI indicated on the $y$-axis. For display purposes, each row is normalized to have the same maximum (white).
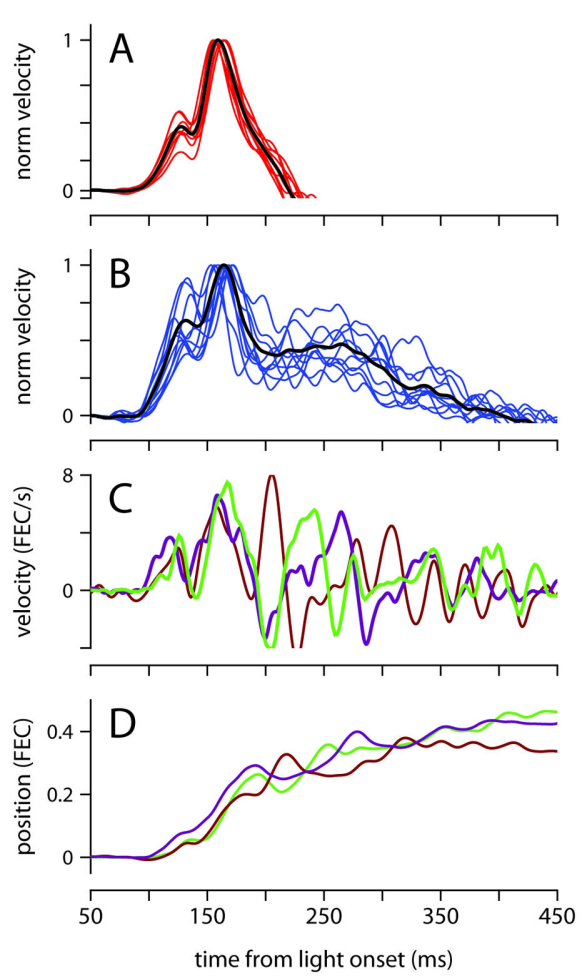

FIGURE 3 | Analysis of velocity peaks. (A) Normalized eyelid velocity averaged over all trials (black), and averaged separately for individual sessions (red) for a mouse trained with a 175-ms, and (B) for a mouse trained with a 400-ms ISI (individual sessions shown in blue).

(C) Eyelid velocity, and (D) eyelid position for three trials taken from the subject in (B). (E) Number of velocity peaks found in single trials between 100 and $200 \mathrm{~ms}$ from light onset, and $(\mathbf{F})$ the interval of time between consecutive velocity peaks. Histograms were computed separately for each
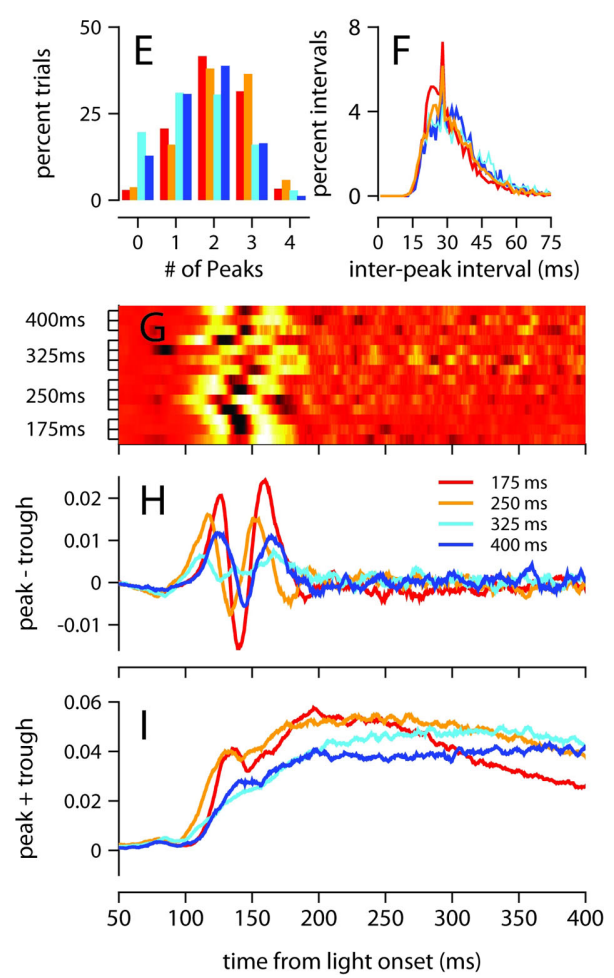

mouse and averaged over ISI condition. (G) Difference between the probability of finding a peak and a trough at each ms from light onset. Each row of the heat map contains the difference in peak and trough probability functions for an individual mouse, with trained ISI indicated on the $y$-axis. For display purposes, each row is converted to a $z$-score. (H) Difference, and (I) sum of probability functions for peaks and troughs, averaged over ISI condition. Color code for (E), (F), (H), and (I) indicates trained ISI and is shown in (H). FEC, fraction eyelid closure. 
remained high after the first $200 \mathrm{~ms}$ (Figures 3C,I), the clustering disappeared (Figures 3G,H), indicating that the location of peaks and troughs is not time-locked and varies from trial to trial during the later part of the eyelid movement.

\section{OSCILLATORY PROPERTIES OF CONDITIONED EYELID MOVEMENTS AT DIFFERENT ISIS}

We now characterize in detail the oscillatory properties of conditioned eyelid movements in single trials, and determine if these properties are differentially modulated as a function of ISI. Figure 3F already hints at an underlying oscillation of $33 \mathrm{~Hz}(30 \mathrm{~ms}$ interpeak-interval) in the first $200 \mathrm{~ms}$ after light onset, but peak detection is not the optimal way to measure the oscillatory properties of eyelid movements because it depends on an arbitrary cutoff threshold, and cannot distinguish between the strength of oscillations at different frequencies (see Materials and Methods). Thus, we follow the example of previous authors (Domingo et al., 1997; Koekkoek et al., 2002), and focus instead on the frequencydomain characteristics revealed by the spectrogram of the eyelid acceleration signal (Figure 4).

The mean power spectrum of acceleration showed a broad peak centered around $30 \mathrm{~Hz}$ for all ISIs (clearly visible in Figure 4, which shows the spectrogram for one of the mice trained with an ISI of $175 \mathrm{~ms}$ ), both during the early part of the eyelid movement (Figure 5A) and also later on (Figure 5B). This is consistent with the idea that regardless of ISI, the eyelid has a tendency to accelerate and decelerate in bursts, oscillating in the $30-\mathrm{Hz}$ frequency range for the entire duration of the movement (Figures $3 \mathrm{C}$ and 4). There was a clear modulation of total power according to the

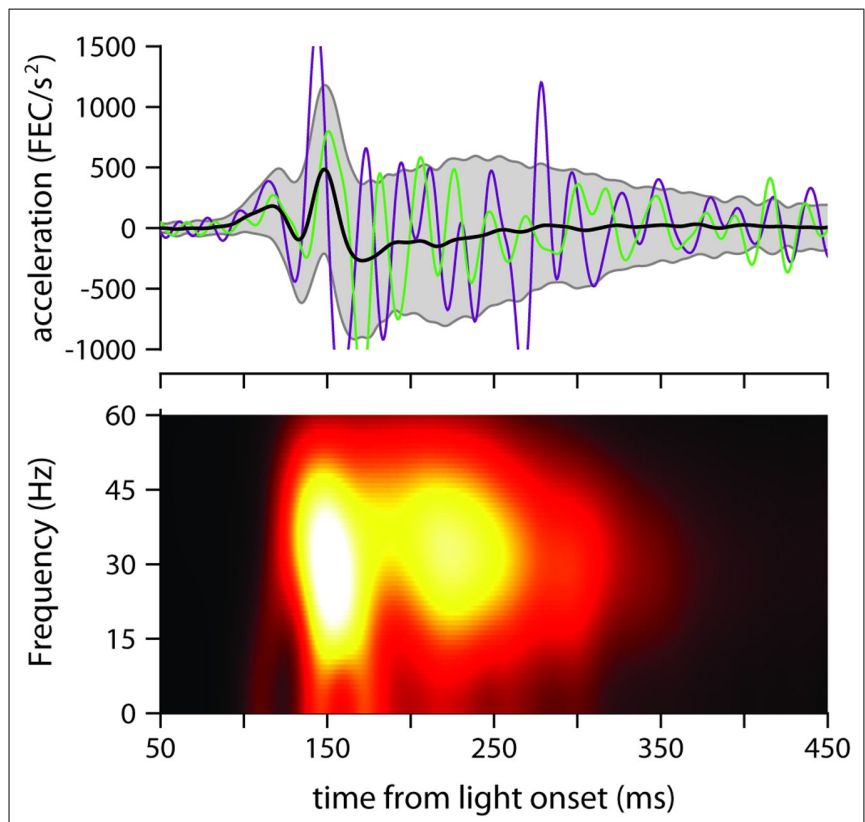

FIGURE 4 | Eyelid acceleration. Top: mean eyelid acceleration (black) \pm 1 SD (gray shaded region), and example acceleration records from two randomly selected trials (green and purple), for one of the mice trained with a 175-ms ISI (same mouse as in Figure 3A). Bottom: mean of all single-trial spectrograms of the eyelid acceleration signal for the same mouse (see Materials and Methods for details about spectrogram generation). trained ISI in the early response period (Figure 5A), which suggests that acceleration bursts during this period are larger for the fast eyelid movements generated at short ISIs, and smaller for the slower eyelid movements at longer ISIs (Figure 1B). However, this straightforward relationship between total power and ISI appeared to dissipate with time and could no longer be detected in the later parts of the eyelid movement (Figure 5B).

Thus, to further evaluate how the frequency and power of oscillations evolve over time, we computed the spectrogram of the acceleration signal throughout the extent of the eyelid movement (Figure 4). For each time point, we determined the frequency at which power was maximal (Figure 5D), and the power at this frequency (Figure 5C). Power in the interval 150-250 ms after light onset decreased as the ISI was lengthened (Figure 5C; all pairwise comparisons $p=0.001)$. As shown in Figure 5D, there was
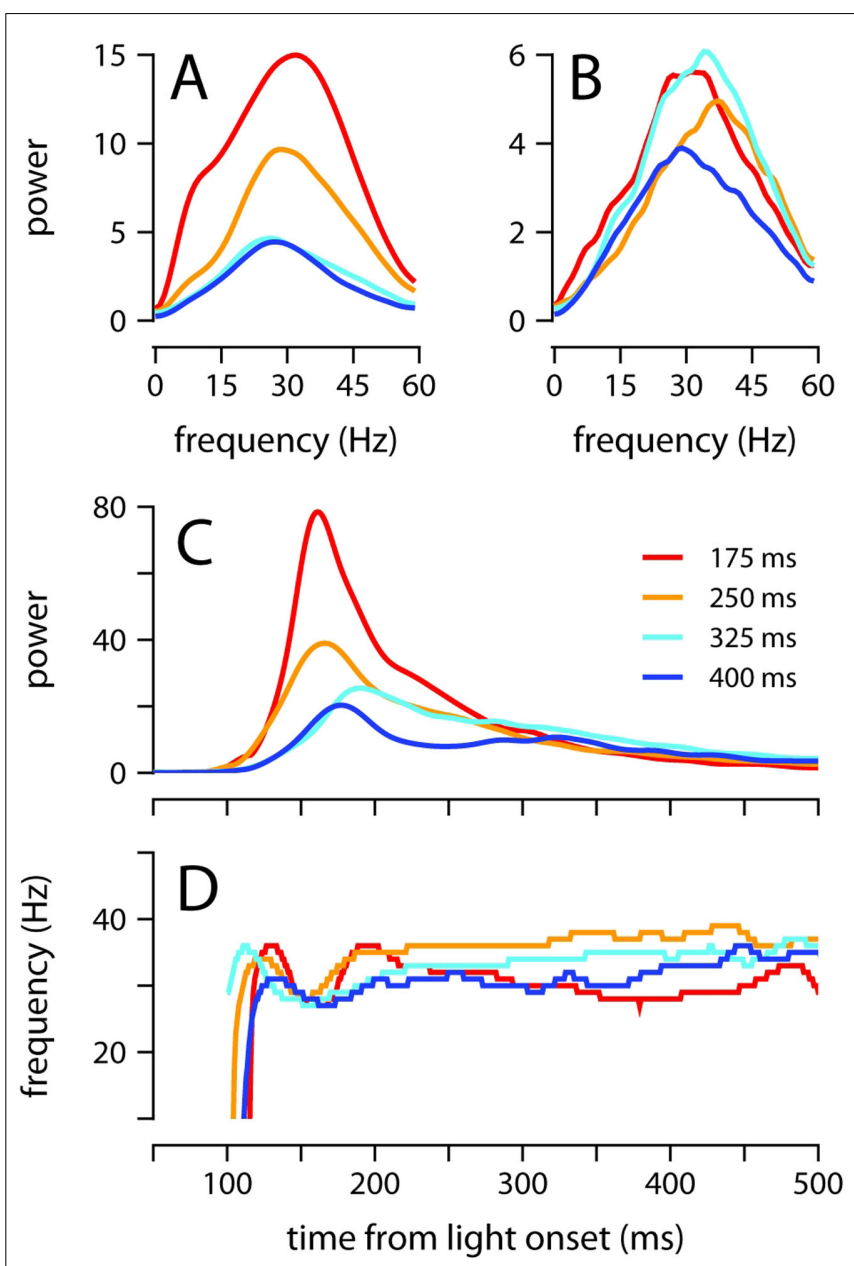

FIGURE 5 | Oscillatory properties of the conditioned eyelid response. (A) Average power spectrum of eyelid acceleration during the first $200 \mathrm{~ms}$, and (B) from 200 to $500 \mathrm{~ms}$ after light onset. Spectra are plotted for each ISI separately. (C) Maximum power, and (D) dominant frequency of oscillation, in the eyelid acceleration signal. The value plotted at each moment in time in (C,D) is computed over a $\sim 50$ ms spectrogram window centered at that moment (see Materials and Methods). Each ISI plotted separately. For all plots, power is in units of (fraction eyelid closure $\left./ \mathrm{s}^{2}\right)^{2} \mathrm{~Hz}^{-1} \times 10^{4}$, and color code indicates trained ISI as shown in (C) 
a small dip in the dominant frequency of the eyelid oscillation at the time of maximum power, but it is clear that for the majority of the eyelid movement the dominant frequency remained within 30-40 Hz with no apparent dependence on ISI (Figure 5D). These results indicate that mice adjust the speed of the conditioned eyelid movement by modulating the amplitude of acceleration bursts, but not the rate at which individual bursts occur.

\section{CONDITIONED EYELID MOVEMENTS ARE NEITHER STARTLE- NOR FIXED SHORT-LATENCY RESPONSES}

Because the onset latency of the conditioned eyelid movement appears relatively fixed and unaffected by ISIs in the range 175-400 (Figures 2A,B), it is reminiscent of startle-reflex and short-latency blinks previously observed during eyelid conditioning in mice trained with an auditory stimulus (Boele et al., 2010). However, the data in Figure $\mathbf{6}$ demonstrate that under certain circumstances, our mice were able to adjust the onset of the movement, which is inconsistent with the known properties of startle and short-latency responses. We trained two mice with an ISI of $100 \mathrm{~ms}$, an interval that is shorter than the latency of the first velocity peak in our experiments (Figure 2B). Both mice showed adaptive timing, achieving maximum eyelid closure $\sim 75 \mathrm{~ms}$ earlier than the mice trained with the 175-ms interval (Figure 6A). Timing was achieved by shifting movement onset and increasing the magnitude of the first velocity peak relative to the second (Figure 6B). The early period with the two time-locked velocity peaks resembled early periods for other ISIs, but appeared shifted slightly earlier in time (Figure 6C). This adaptive shift in onset latency and the profile of response contrasts with the fixed onset latency and inflexible trajectory of startle and short-latency responses in previous studies that have used an auditory stimulus instead of a light (Boele et al., 2010).

Startle, short-latency and conditioned eyelid responses can also be distinguished with regards to their development during training (Boele et al., 2010). Startle-responses are small non-associative blinks that can be detected from the very first presentation of the CS before any air-puffs have been given, and short-latency responses are learned very quickly, typically within the first session of conditioning ( $<100$ trials). In contrast, Figure 7 shows that conditioned eyelid movements in our mice developed very gradually and took many sessions to appear. Furthermore, when conditioned eyelid movements were first observed, mean behavior did not immediately exhibit the characteristic "two-peak" velocity profile found at the completion of training (compare yellow and brown traces in Figure 7). Instead, the conditioned eyelid movements acquired their characteristic shape over weeks of conditioning, unlike startle- and short-latency blinks, whose kinematic profiles and onset latencies remain constant from the beginning (Boele et al., 2010).

\section{COMPARISON BETWEEN MDMT AND HIGH-SPEED VIDEO RECORDING}

To rule out the possibility that our results are an artifact of the MDMT technology that we have used to monitor eyelid position (Koekkoek et al., 2002), we developed a high-speed video capture system and recorded conditioned eyelid movements in one of the 250-ms mice with both systems simultaneously. There were some minor differences between the two methods, particularly

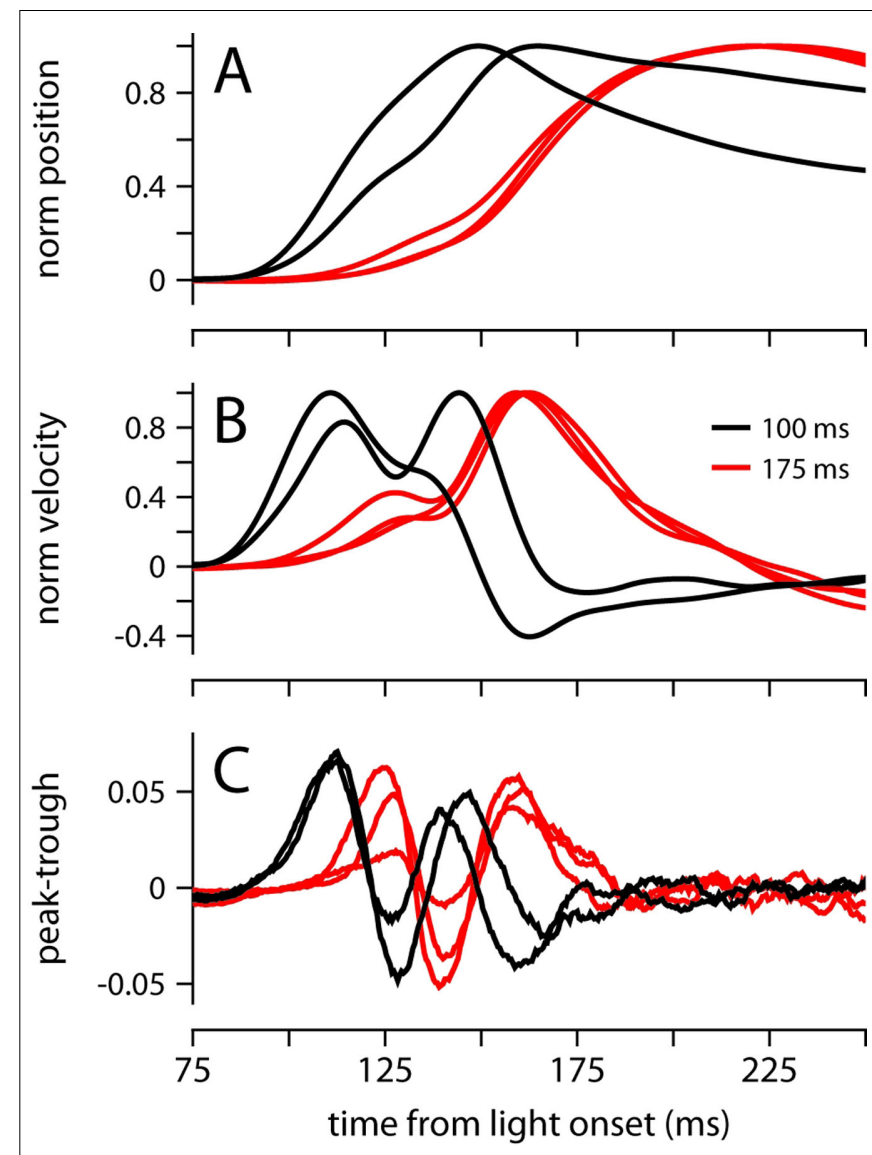

FIGURE 6 | Conditioned eyelid movements with a 100-ms ISI. (A) Normalized average eyelid position, and (B) normalized average velocity for mice trained with a 100-ms (black) or $175 \mathrm{~ms}$ ISI (red). (C) Difference between the probability of finding a peak and a trough at each $\mathrm{ms}$ from light onset. Color code for all plots indicates ISI and is shown in (B).

with regards to the precise eyelid position around the time of maximum eyelid closure (see Materials and Methods); however, all major findings from the preceding analysis were verified by the video data: maximum eyelid closure occurred at the same time (Figure 8A, MDMT: $268 \pm 75 \mathrm{~ms}$, Video: $279 \pm 78 \mathrm{~ms}$ ), the characteristic two-peaked velocity profile was observed (Figures 8B,C), and acceleration power spectrums largely overlapped with a peak around $30 \mathrm{~Hz}$ (Figure 8D). Finally, we also used video to record eyelid movements in a session without the magnet glued on the eyelid (no MDMT signal) and found that the general properties of the data were unchanged (data not shown).

\section{DISCUSSION}

We have shown that mice trained with different ISIs can learn to time eyelid movements appropriately. Our data indicate that for ISIs in the 175-400-ms range, mice achieve precise timing by modulating the velocity of the blink but not its onset, in a manner that is incompatible with the timescale-invariant hypothesis. Analysis of movement kinematics in individual trials reveals the details of how this high-level control strategy is implemented by the brain: (1) conditioned eyelid movements are composed of discrete bursts 

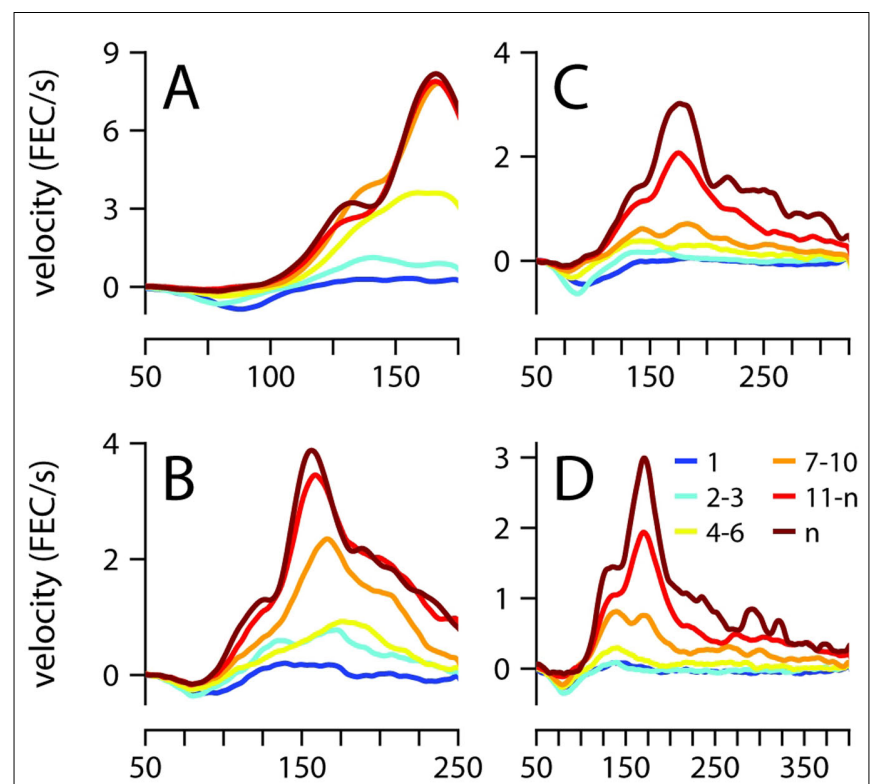

time from light onset (ms)

FIGURE 7 | Acquisition of the conditioned eyelid response. (A) Eyelid velocity averaged across different sessions during the training phase, for mice trained with ISls of $175 \mathrm{~ms}$ (B) $250 \mathrm{~ms}$, (C) $325 \mathrm{~ms}$, and (D) $400 \mathrm{~ms}$.

The sessions that were used to compute each average are indicated by the color code shown in (D), where $\mathrm{n}$ represents number of the last training session for each mouse. FEC, fraction eyelid closure.

that occur at a dominant frequency of $\sim 30 \mathrm{~Hz}$, (2) the first two bursts of movement are time-locked to the light onset, whereas the precise timing of subsequent bursts is less consistent from trial to trial, (3) maximum eyelid velocity is inversely related to the ISI, and is usually reached during the second burst of the movement, (4) eyelid velocity is controlled by adjusting the amplitude of bursts, but not the frequency at which they are generated.

We decided to do this study in mice because we wanted to open up the door for future investigations using genetic tools not available in other species. But mouse behavior is notorious for being finicky and unstable during eyelid conditioning (Vogel et al., 2002; Boele et al., 2010), and for this reason it may seem surprising that we were able to detect changes in the eyelid movement that were often on the order of just a few tens of milliseconds. Before we discuss the implications of our findings for the neural control of movement, we summarize some of the key methodological advances that have made our work possible.

\section{A NEW EXPERIMENTAL APPROACH TO STUDY ADAPTIVE TIMING IN MICE}

We have introduced a number of technical and procedural modifications to improve performance and to help isolate the adaptively timed component of the conditioned eyelid movement: (1) we have used low-intensity light as the CS because previous work has shown that in mice, the eyelid response to the more traditional tone stimulus includes a non-associative auditory startle-reflex that complicates the analysis of onset latency and may interfere with the production of the conditioned eyelid movement (Vogel
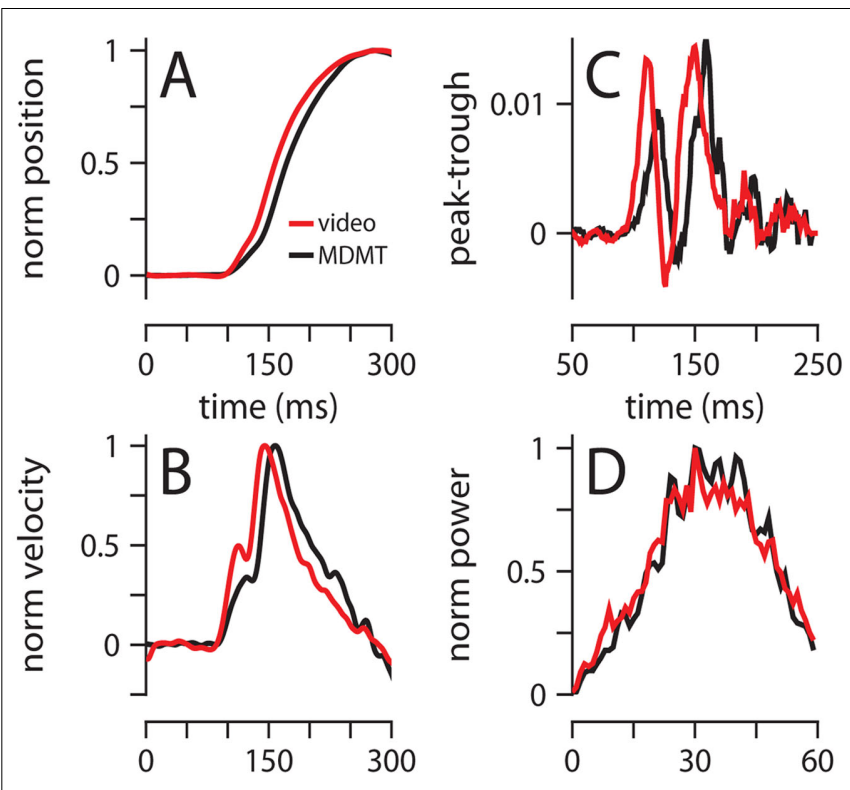

time (ms)

freq $(\mathrm{Hz})$

FIGURE 8 | Comparison of video and MDMT. (A) Normalized average position, and (B) normalized average velocity. (C) Difference between the probability of finding a peak and a trough at each millisecond from light onset. (D) Normalized average power spectrum of eyelid acceleration. Color code for all plots indicates the type of recording system and is shown in (A). All data is from two 300-trial sessions with a mouse trained with a 250-ms ISI.

et al., 2002; Boele et al., 2010). (2) We have used a very mild air-puff because it has been suggested recently that the much stronger periorbital stimulation typically employed for eyeblink conditioning in mice leads to a fear-related blink with a relatively fixed short-latency (Boele et al., 2010), and there is indirect evidence that in some cases this short-latency response can interfere with the adaptively timed component of the conditioned eyelid response (Aiba et al., 1994; Sakamoto and Endo, 2011). (3) We have developed a system that allows mice to be conditioned while they are actively engaged in one of their favorite activities: treadmill walking. Maintaining activity levels high helps minimize the otherwise frequent periods of "quiet wakefulness" that are partly responsible for the low levels of conditioning observed in previous studies (Boele et al., 2010). Indeed, all our mice performed at a very high level throughout the testing phase (Table 1), which included many sessions, and many trials in each session. This excellent performance, together with the lack of startle or fear-related short-latency eyelid responses, and the advantages of a head-fixed preparation, make our cylindrical treadmill system ideally suited for future studies aimed at investigating the neural mechanisms underlying the adaptive timing of movement.

\section{MOTONEURON CONTROL OF MOVEMENT SPEED: A HYPOTHESIS}

Electromyographic recordings from blink-related muscles like the orbicularis oculi can help us understand how the brain controls eyelid movement. As already noted, blinks are made by accelerating and decelerating the eyelid in brief bursts that can be identified in individual trials as velocity peaks in the eyelid movement. Previous 
work has established that these peaks of velocity are correlated with peaks in the orbicularis oculi EMG signal (Trigo et al., 1999; Gruart et al., 2000; Ivarsson and Svensson, 2000), a finding that is consistent with earlier work demonstrating that there is a tight relationship between the maximum velocity of the eyelid during reflex blinks and the peak of the EMG response (Evinger et al., 1991; Gruart et al., 1995). Because EMG signals sum the action potentials that occur in a large population of motoneurons, the amplitude of a velocity peak is thought to reflect the number of motoneurons that are synchronously activated at that time (Evinger et al., 1991).

Given these considerations, our behavioral findings suggest a particular mechanism by which motoneuron firing could control the velocity of the conditioned eyelid response, and thus help adjust the time of maximum eyelid closure: when the ISI is short, velocity peaks are large and movement is fast because there is a large number of motoneurons that have been recruited and fire synchronously at the dominant frequency of $\sim 30 \mathrm{~Hz}$. When the ISI is longer, less motoneurons are active, but those recruited still fire at $\sim 30 \mathrm{~Hz}$, which helps explain why the interval between velocity peaks remains the same regardless of ISI. Since a smaller fraction of the motor pool is active, velocity peaks are smaller and the resulting movement is slower.

Similar versions of this recruitment strategy have been proposed for linearizing the plant during blink-related movements of the nictitating membrane in rabbits (Lepora et al., 2007, 2009; Mavritsaki et al., 2007), raising the possibility that recruitment could play a similar role in the eyelid system. We note that in other animal species, the frequency at which the eyelid oscillates is different from $\sim 30 \mathrm{~Hz}$, and it appears to be related to body weight (Gruart et al., 2000). However, there is no reason why the mechanisms we have suggested here for mice could not be applied to motor control in other animals as well. The key feature of the "recruitment-only" strategy proposed above is that adaptive timing is achieved by dynamically regulating the number of motor units that contribute to the movement rather than their individual firing rates.

\section{ORIGIN OF THE OSCILLATORY CONTROL SIGNAL}

It is known that the periodic peaks of velocity in the conditioned eyelid response are caused by oscillatory bursts of EMG activity in the orbicularis oculi (Gruart et al., 1995, 2000; Trigo et al., 1999; Ivarsson and Svensson, 2000); what is yet to be determined are the neural mechanisms that give rise to the oscillation in the EMG signal in the first place. Some authors have suggested that intrinsic properties of facial motoneurons may be responsible for generating oscillatory bursts in the orbicularis oculi muscle (Trigo et al., 1999). The general idea behind this proposal is that because blink-related motoneurons have conductances that cause spike after-hyperpolarizations with a duration that matches the period of the EMG oscillation (Baker et al., 1980; Fanardjian et al., 1983), they may be able to generate rhythmic output at approximately the right frequency. However, it is worth noting that the interval between velocity peaks remained the same for all conditions, and across the entire duration of movement, even though the velocity of the movements varied greatly. It seems unlikely that intrinsic membrane conductances could continue to generate an output signal at the same frequency despite receiving input signals varying in strength over several orders of magnitude. Furthermore, even if intrinsic properties allow individual motoneurons to fire rhythmically, these properties alone cannot explain why the activity of the population is synchronized at a particular frequency during movement.

Our finding that the first two velocity peaks of the conditioned eyelid response are time-locked indicates that orbicularis oculi motoneurons become synchronized with remarkable temporal precision at the beginning of the movement, suggesting that they may receive common rhythmic drive from upstream areas. The cerebellar interpositus nucleus (CIN) is particularly well-suited for providing this drive during the generation of the conditioned eyelid response because: (1) it projects to orbicularis oculi motoneurons, both monosynaptically and via the red nucleus (Fanardjian and Manvelyan, 1984; Morcuende et al., 2002), (2) it is necessary for generating the conditioned eyelid response (McCormick and Thompson, 1984; Chen et al., 1996), (3) if stimulated artificially, it generates eyelid movements with velocity peaks occurring at the frequency of stimulation (Jimenez-Diaz et al., 2004), and (4) it contains neurons whose firing oscillates and tracks the rhythmic bursts in the EMG of the orbicularis oculi and the velocity peaks of the conditioned eyelid response (Sanchez-Campusano et al., 2007). The specific role of the CIN remains an open question, however, and it has been suggested that its activity may be used to modulate rather than drive the eyelid movements (Delgado-Garcia and Gruart, 2002; Sanchez-Campusano et al., 2009). Future studies will aim to define the function of the CIN and understand the role that it plays in the different aspects of the control strategy for adaptive timing that we have uncovered here.

The same strategy may be used in other movements whose timing is under cerebellar control. For example, much like eyeblink conditioning (Medina et al., 2000), the execution and adaptation of saccades requires the cerebellum (Hopp and Fuchs, 2004; Catz and Thier, 2007). It is intriguing that saccade velocity trajectories are strikingly similar to the eyelid trajectories analyzed here, revealing a fixed-duration acceleration phase followed by a deceleration phase which is lengthened or shortened for longer or shorter saccade durations (Van Opstal and Van Gisbergen, 1987). Understanding why such a peculiar control strategy is utilized may provide some clues about the rules and goals governing information processing in the cerebellum.

\section{MATERIALS AND METHODS \\ ANIMALS}

Sixteen C57BL/6J mice (Mus musculus), acquired from The Jackson Laboratory, were used as subjects. Animals were kept on a 12:12-h light/dark cycle, set for 7 a.m.-7 p.m. darkness so that all experiments took place during the dark period. Mice were between the ages of 10 and 13.5 weeks before surgery and had not been used in any prior experiments. To prepare the animals to be head-fixed, mice were anesthetized with isoflurane and placed in a stereotaxic apparatus. Two screws were inserted into the surface of the skull, and a custom-cut rectangular metal head plate was cemented to the screws and skull using C\&B-Metabond ${ }^{\circledR}$. After allowing $3+$ days for recovery from surgery, mice were then habituated to being placed in the experimental apparatus for 3-5 days before 
training began. All procedures had been approved in advance by the Institutional Animal Care and Use Committee at The University of Pennsylvania and were in accordance with the NIH Guide for the Care and Use of Laboratory Animals.

\section{EXPERIMENTAL APPARATUS, STIMULUS CONTROL AND DATA ACQUISITION}

All our experiments were done in head-fixed mice that were placed on top of a "cylindrical treadmill" and allowed to walk on top of it. An Exervo TeraNova ${ }^{\mathrm{TM}} \mathrm{EVA}$ foam roller with a diameter of

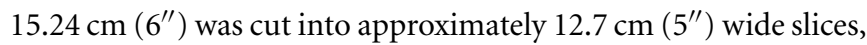
and a hole was drilled through the axis of the resulting cylinder, in order to mount the cylinder on a horizontal pole outfitted with ball bearings. This freely rotating cylinder was then positioned below a custom-built head-fixing device onto which the mouse's head plate could be mounted. The entire experimental apparatus was placed within a soundproof box (Med Associates, Inc.), and kept in the dark.

TDT System 3 processors were used to control the timing of stimuli, and to acquire the eyelid signal at a sampling rate of $2034.5 \mathrm{~Hz}$. The unconditioned stimulus was a nitrogen air-puff (80 psi, 20 ms duration) controlled by an API MPPI-3 pressure injector, and delivered via a 27.5 gauge needle positioned $\sim 1 \mathrm{~cm}$ from the subject's left cornea. Because inherent delays in the electronics, and the time it takes for the air to travel from the pressure injector to the mouth of the needle, the air-puff hits the cornea 12$15 \mathrm{~ms}$ after the stimulus is triggered. All our mice were successfully conditioned even though the air-puff stimulation was relatively mild and its perceived intensity may have been reduced even further during a conditioned response, when the eyelid is partially closed and protecting the cornea. In this regard, as with all previous studies using air-puff stimulation, the learned eyelid response is likely to be the result of both classical and instrumental/operant conditioning processes. The CS was a blue LED, positioned $2-3 \mathrm{~cm}$ directly in front of the subject. Because the experimental box was kept dark, the blue light is a salient stimulus easily detectable by both eyes. During a conditioned response, however, the eyelids are partially closed, and in theory this could interfere with the detection and processing of the light stimulus. No attempt was made to examine this potential interference, or to investigate how the properties of the conditioned eyelid movement differ when the light is presented exclusively to the left or right eye. Nevertheless, the very high-levels of performance in all the mice (Table 1) indicate that if present, interference due to partially closed eyelids is minimal. Furthermore, our results examining the kinematic properties of the eyelid movement at different ISIs clearly demonstrate that light stimuli in our eyeblink conditioning task are well-suited for investigating adaptive timing of motor behavior in mice.

We used the MDMT method commercially available from www.neurasmus.com to measure movement of the left eyelid (ipsilateral to the air-puff stimulation; Koekkoek et al., 2002): before each conditioning session, mice were briefly anesthetized with isoflurane and a small neodymium magnet was attached to the left lower eyelid with cyanoacrylate ("Super Glue ${ }^{\mathrm{TM}}$ "). An NVE GMR magnetometer positioned above the upper left eyelid was used to detect the movement of the magnet and obtain an electrical signal proportional to lower eyelid position. In a few experiments, we also performed high-speed video recording of eyelid movements, using an AVT GE680 monochromatic camera to monitor a $256 \times 256$ pixel region (approximately the smallest rectangle which completely enclosed the eye) at $350 \mathrm{fps}$. Lighting was provided by an infrared illuminator. Video capture was controlled via customwritten code in Matlab (The Mathworks, Natick, MA, USA). Small differences between video and MDMT signals were the result of a non-linearity in the NVE chip that generates exponentially bigger signals for any given eyelid displacement as the small neodymium magnet gets closer to the magnetometer.

\section{DESIGN}

Each mouse was assigned an ISI prior to training. Experiments consisted of three phases: 3 days of habituation, followed by 14-15 daily training sessions, and 7-10 daily test sessions. For each habituation session, mice were placed on top of the cylindrical treadmill with the head-fixed for 30-40 min, but no stimuli were presented. Daily conditioning sessions during the training phase consisted of 100 paired presentations of CS and US, separated by the assigned ISI. Learning proceeded gradually, and performance (as measured by percent of trials with a conditioned eyelid response) typically stabilized after 10 days of training. Training in the two mice with the lowest performance in the 400-ms ISI group was extended to 22 sessions, but no improvements were observed. During the testing phase, sessions began with 5-7 US-alone trials, used to calibrate our measurements. This was followed by 200-300 trials, half paired presentations at the same ISI used during training, and half CS-alone trials, presented in alternating order (except two mice, with $175 \mathrm{~ms}$ ISI, for whom this order was randomized). The intertrial interval was set according to the following constraints: at least $10 \mathrm{~s}$ had to elapse, the eyelid had to be open below a predetermined threshold, and eyelid position had to be stable for at least $1 \mathrm{~s}$ for a trial to begin. Threshold and stability parameters were adjusted for each session by the experimenter. Experiments were performed at approximately the same time of day for each mouse.

\section{ANALYSIS}

Eyelid data was imported into Matlab and filtered in the forward and reverse direction with a fifth-order low-pass Butterworth filter and a cutoff frequency at $60 \mathrm{~Hz}$. We verified that similar velocity peaks were found on the mean of the filtered and un-filtered signal, and that changing the order or cutoff frequency of our filter did not drastically alter acceleration power spectra. The signal was calibrated for each session so that the size of a full blink was 1, and eyelid position in the 100 -ms preceding each trial was subtracted from that trial, resulting in all trials beginning from a position of 0 . For video recording data, the area of eye exposed was calculated in each frame by thresholding the gray scale image and summing the number of pixels in the low-intensity "eye-region" of the resulting image. This signal was calibrated in the same manner as the MDMT data.

All data analysis was done on trials with a conditioned eyelid response. An eyelid movement was counted as a conditioned response if the eyelid displacement in a CS-alone trial exceeded $10 \%$ of full closure within $500 \mathrm{~ms}$ of light onset. Probability density functions of position and velocity maxima (Figures 2C,D) were generated by smoothing raw data 
with a gaussian kernel, using the Matlab function ksdensity. Local peak detection was performed using the freely available program peakdet (http://billauer.co.il/peakdet.html). Peak and trough probability were computed for each sample by dividing the number of peaks or troughs found over all response trials at each sample by the total number of trials. Differences or sums of peak and trough probabilities were then smoothed with a 10 -ms-wide running average. For display purposes in Figure 3G, the peaktrough probability difference for each mouse was standardized to a $z$-score. Spectrograms were computed using the Matlab function spectrogram: the acceleration signal was divided into overlapping $49.15 \mathrm{~ms}$ sections, with $0.98 \mathrm{~ms}$ increments between windows, and each section was smoothed with a hamming window. The power spectrum of each segment was then computed as the square of the

\section{REFERENCES}

Aiba, A., Kano, M., Chen, C., Stanton, M. E., Fox, G. D., Herrup, K., Zwingman, T. A., and Tonegawa, S. (1994). Deficient cerebellar long-term depression and impaired motor learning in mGluR1 mutant mice. Cell 79, 377-388.

Baker, R., McCrea, R. A., and Spencer, R. F. (1980). Synaptic organization of cat accessory abducens nucleus. $J$. Neurophysiol. 43, 771-791.

Boele, H. J., Koekkoek, S. K., and De Zeeuw, C. I. (2010). Cerebellar and extracerebellar involvement in mouse eyeblink conditioning: the ACDC model. Front. Cell. Neurosci. 3:19. doi:10.3389/neuro.03.019.2009

Boneau, C. A. (1958). The interstimulus interval and the latency of the conditioned eyelid response. J. Exp. Psychol. 56, 464-471.

Catz, N., and Thier, P. (2007). Neural control of saccadic eye movements. Dev. Ophthalmol. 40, 52-75.

Chen, L., Bao, S., Lockard, J. M., Kim, J. K., and Thompson, R. F. (1996). Impaired classical eyeblink conditioning in cerebellarlesioned and Purkinje cell degeneration (pcd) mutant mice. J. Neurosci. 16, 2829-2838.

Delgado-Garcia, J. M., and Gruart, A. (2002). The role of interpositus nucleus in eyelid conditioned responses. Cerebellum 1, 289-308.

Domingo, J. A., Gruart, A., and Delgado-Garcia, J. M. (1997). Quantal organization of reflex and conditioned eyelid responses. J. Neurophysiol. 78, 2518-2530.

Evinger, C., Manning, K. A., and Sibony, P. A. (1991). Eyelid movements. Mechanisms and normal data. Invest. Ophthalmol. Vis. Sci.32, 387-400.

Fanardjian, V. V., and Manvelyan, L. R. (1984). Peculiarities of cerebellar excitation of facial nucleus motoneurons. Neurosci. Lett. 49, 265-270.

Fanardjian, V. V., Manvelyan, L. R., and Kasabyan, S. A. (1983). Mechanisms regulating the activity of facial nucleus motoneurones - 1 . Antidromic activation. Neuroscience 9, 815-822.

Fortenbaugh, D. M. (2011). The biomechanics of the baseball swing. Open Access Dissertations 540, 1-235.

Freeman, J. H. Jr., Nicholson, D. A., Muckler, A. S., Rabinak, C. A., and DiPietro, N. T. (2003). Ontogeny of eyeblink conditioned response timing in rats. Behav. Neurosci. 117, 283-291.

Gentner, D. R. (1987). Timing of skilled motor performance: tests of the proportional duration model. Psychol. Rev. 94, 255.

Gormezano, I., Kehoe, E. J., and Marshall, B. S. (1983). Twenty years of classical conditioning research with the rabbit. Prog. Psychobiol. Physiol. Psychol. 10, 197-275.

Gray, R. (2002). Behavior of college baseball players in a virtual batting task. J. Exp. Psychol. Hum. Percept. Perform. 28, 1131.

Grossberg, S., and Schmajuk, N. A. (1989). Neural dynamics of adaptive timing and temporal discrimination during associative learning. Neural Netw. 2, 79-102.

Gruart, A., Blazquez, P., and DelgadoGarcia, J. M. (1995). Kinematics of spontaneous, reflex, and conditioned eyelid movements in the alert cat. J. Neurophysiol. 74, 226-248.

Gruart, A., Schreurs, B. G., del Toro, E. D., and Delgado-Garcia, J. M. (2000). Kinetic and frequencydomain properties of reflex and conditioned eyelid responses in the rabbit. J. Neurophysiol. 83, 836-852.

Hopp, J. J., and Fuchs, A. F. (2004). The characteristics and neuronal substrate of saccadic eye movement plasticity. Prog. Neurobiol. 72, 27-53.

absolute value of the short-time Fourier transform of each segment. For all power spectral analyses, spectra were first computed for individual trials, averaged over all trials within a mouse, then averaged across mice within condition.

\section{ACKNOWLEDGMENTS}

We are grateful to M. Moser for technical assistance, and to R. Lee, S. Martin, and J. Andrews-Labenski for help with machining and electrical design. C. De Zeeuw, H. J. Boele, and S. Koekkoek were instrumental in helping us set up the MDMT system, and S. Wang, I. Ozden, A. Giovannucci, and F. Najafi helped develop the original "floating ball" apparatus for eyeblink conditioning on which our "rotating treadmill" is based. Work supported with funds from a Searle Scholars Foundation grant to Javier F. Medina.

Ivarsson, M., and Svensson, P. (2000). Conditioned eyeblink response consists of two distinct components. J. Neurophysiol. 83, 796-807.

Jimenez-Diaz, L., Navarro-Lopez Jde, D., Gruart, A., and Delgado-Garcia, J. M. (2004). Role of cerebellar interpositus nucleus in the genesis and control of reflex and conditioned eyelid responses. J. Neurosci. 24, 9138-9145.

Koekkoek, S. K., Den Ouden, W. L., Perry, G., Highstein, S. M., and De Zeeuw, C. I. (2002). Monitoring kinetic and frequency-domain properties of eyelid responses in mice with magnetic distance measurement technique. J. Neurophysiol. $88,2124-2133$.

Koekkoek, S. K., Hulscher, H. C., Dortland, B. R., Hensbroek, R. A., Elgersma, Y., Ruigrok, T. J., and De Zeeuw, C. I. (2003). Cerebellar LTD and learning-dependent timing of conditioned eyelid responses. Science 301, 1736-1739.

Lepora, N. F., Mavritsaki, E., Porrill, J., Yeo, C. H., Evinger, C., and Dean, P. (2007). Evidence from retractor bulbi EMG for linearized motor control of conditioned nictitating membrane responses. J. Neurophysiol. 98, 2074-2088.

Lepora, N. F., Porrill, J., Yeo, C. H., Evinger, C., and Dean, P. (2009). Recruitment in retractor bulbi muscle during eyeblink conditioning: EMG analysis and commondrive model. J. Neurophysiol. 102, 2498-2513.

Levey, A. B., and Martin, I. (1968). Shape of the conditioned eyelid response. Psychol. Rev. 75, 398-408.

Marquis, D., and Porter, Jr. J. (1939). Differential characteristics of conditioned eyelid responses established in reflex and voluntary reinforcement. J. Exp. Psychol. 24, 347-365.

Mauk, M. D., and Ruiz, B. P. (1992). Learning-dependent timing of Pavlovian eyelid responses: differential conditioning using multiple interstimulus intervals. Behav. Neurosci. 106, 666-681.

Mavritsaki, E., Lepora, N., Porrill, J., Yeo, C. H., and Dean, P. (2007). Response linearity determined by recruitment strategy in detailed model of nictitating membrane control. Biol. Cybern. 96, 39-57.

McCormick, D. A., and Thompson, R. F. (1984). Cerebellum: essential involvement in the classically conditioned eyelid response. Science 223, 296-299.

Medina, J. F., Nores, W. L., Ohyama, T., and Mauk, M. D. (2000). Mechanisms of cerebellar learning suggested by eyelid conditioning. Curr. Opin. Neurobiol. 10, 717-724.

Morcuende, S., Delgado-Garcia, J. M., and Ugolini, G. (2002). Neuronal premotor networks involved in eyelid responses: retrograde transneuronal tracing with rabies virus from the orbicularis oculi muscle in the rat. J. Neurosci. 22, 8808-8818.

Sakamoto, T., and Endo, S. (2011). Amygdala, deep cerebellar nuclei and red nucleus contribute to delay eyeblink conditioning in C57BL/6 mice. Eur. J. Neurosci. 32, 1537-1551.

Sanchez-Campusano, R., Gruart, A., and Delgado-Garcia, J. M. (2007). The cerebellar interpositus nucleus and the dynamic control of learned motor responses. J. Neurosci. 27, 6620-6632.

Sanchez-Campusano, R., Gruart, A., and Delgado-Garcia, J. M. (2009). Dynamic associations in the cerebellar-motoneuron network during motor learning. J. Neurosci. 29, 10750-10763.

Schmidt, R. A., and Lee, T. D. (2005). Motor Control and Learning: A Behavioral Emphasis. Champaign, IL: Human Kinetics. 
Trigo, J. A., Gruart, A., and DelgadoGarcia, J. M. (1999). Discharge profiles of abducens, accessory abducens, and orbicularis oculi motoneurons during reflex and conditioned blinks in alert cats. J. Neurophysiol. 81, 1666-1684.

Van Der Giessen, R. S., Koekkoek, S. K., van Dorp, S., De Gruijl, J. R., Cupido, A., Khosrovani, S., Dortland, B., Wellershaus, K., Degen, J., Deuchars, J., Fuchs, E. C., Monyer, H., Willecke, K., De Jeu, M. T., and De Zeeuw, C. I. (2008). Role of olivary electrical coupling in cerebellar motor learning. Neuron 58, 599-612.

Van Opstal, A. J., and Van Gisbergen, J. A. (1987). Skewness of saccadic velocity profiles: a unifying parameter for normal and slow saccades. Vision Res. 27, 731-745.

Vogel, R. W., Ewers, M., Ross, C., Gould, T. J., and Woodruff-Pak, D. S. (2002). Age-related impairment in the 250-millisecond delay eyeblink classical conditioning procedure in C57BL/6 mice. Learn. Mem. 9, 321-336.
Williams, T., and Underwood, J. (1986). The Science of Hitting. New York: Simon and Schuster.

Conflict of Interest Statement: The authors declare that the research was conducted in the absence of any commercial or financial relationships that could be construed as a potential conflict of interest.

Received: 13 July 2011; accepted: 11 October 2011; published online: 30 November 2011.
Citation: Chettih SN, McDougle SD, Ruffolo LI and Medina JF (2011) Adaptive timing of motor output in the mouse: the role of movement oscillations in eyelid conditioning. Front. Integr. Neurosci. 5:72. doi: 10.3389/fnint.2011.00072 Copyright (C) 2011 Chettih, McDougle, Ruffolo and Medina. This is an openaccess article subject to a non-exclusive license between the authors and Frontiers Media SA, which permits use, distribution and reproduction in other forums, provided the original authors and source are credited and other Frontiers conditions are complied with. 